\title{
WUIBRAGEC

\section{DESAFIOS PARA A IMPLANTAÇÃO DO BUILDING INFORMATION MODELING EM CURSOS DE TECNOLOGIA EM CONSTRUÇÃO DE EDIFÍCIOS ${ }^{1}$}

\author{
SATO, Mychelle Fabiane Carvalho Bueno (1); BRANDSTETTER, Maria Carolina \\ Gomes de Oliveira (2) \\ (1) Universidade Federal de Goiás, mychellesato@ discente.ufg.br (2) Universidade Federal de \\ Goiás, maria_carolina_brandstetter@ufg.br
}

\begin{abstract}
RESUMO
A demanda do mercado por profissionais capacitados em produzir modelos de informação da construção, extrair dados, detectar interferências entre projetos, gerenciar edifícios após uso e outras operações do Building Information Modeling tem sido um estímulo ao aprimoramento do processo ensino-aprendizado quanto a esta nova filosofia de projetar e construir. Entretanto, ainda é um desafio a inserção do BIM em cursos de tecnologia, e deve-se a isso a relevância desta pesquisa. Esse estudo apresenta a percepção dos alunos em um curso de tecnologia da construção, ofertado no Estado do MT, quanto às dificuldades no aprendizado do BIM, além de sugerir melhorias na metodologia de ensino. As análises abrangeram o BIM como auxílio da compreensão de outras disciplinas e o detalhamento dos tópicos de maior dificuldade no processo de modelagem. Os resultados apontam diretrizes de reestruturação curricular, indicando a partir da matriz do curso, disciplinas que podem utilizar o ensino de BIM e outras que podem utilizar modelagens paramétricas em BIM para apoio didático, além da integração de ações de pesquisa e extensão. Busca-se estimular o meio acadêmico de modo a ampliar as possibilidades de atuação profissional no contexto da Indústria 4.0 e como estratégia fundamental para o desenvolvimento tecnológico deste setor.
\end{abstract}

Palavras-chave: Modelagem da Informação da Construção, Processo Ensino-Aprendizagem, Tecnologia.

\section{ABSTRACT}

The market demand for professionals trained in producing construction information models, extracting data, detecting interference between projects, managing buildings after use and other operations of Building Information Modeling has been a stimulus for teaching-learning process improvement regarding this new philosophy to design and build. However, the inclusion of BIM in technology courses is still a challenge, and this is due to the relevance of this research. This study presents the students perception in a construction technology course, offered in the State of Mato Grosso, regarding the difficulties in learning $B I M$, in addition to suggesting improvements in the teaching methodology. The analyzes covered BIM as an aid to the understanding of other disciplines and the detailing of the most difficult topics in the modeling process. The results point to curricular restructuring guidelines, indicating from the course matrix, disciplines that can use BIM teaching and others that can use parametric models in BIM for didactic support, in addition to the integration of research and extension actions. It seeks to stimulate the academic environment in order to expand the possibilities of professional performance in the context of Industry 4.0 and as a fundamental strategy for the technological development of this sector.

Keywords: Building Information Modeling, Teaching-Learning Process, Technology.

${ }^{1}$ SATO, M. F. C. B.; BRANDSTETTER, M. C. G. de O. Desafios para a implantação do Building Information Modeling em cursos de tecnologia em construção de edifícios. In: SIMPÓSIO BRASILEIRO DE GESTÃ̃ E ECONOMIA DA CONSTRUÇÃO, 12., 2021, Maceió. Anais[...] Porto Alegre: ANTAC, 2021. p.1-8. Disponível em:

https://eventos.antac.org.br/index.php/sibragec/article/view/551. Acesso em: 2 out. 2021. 


\section{INTRODUÇÃO}

Entre os questionamentos que conduzem os educadores na área de ensino de tecnologia das construções a se moldarem diante das necessidades das indústrias 4.0, também chamada de quarta revolução industrial, encontra-se o desafio da implantação de novas formas de trabalho multidisciplinares e colaborativas, que permitam testar alternativas, parametrizar dados e viabilizar decisões de projeto mais racionais e sustentáveis.

O Building Information Modeling (BIM) pode ser definido como uma filosofia de trabalho que agrega profissionais de Arquitetura, Engenharia e Construção (AEC) na elaboração de modelos virtuais gerando uma base de dados com informações de objetos com todos os dados necessários para orçamento, cálculo energético e previsão das fases da construção, entre outras atividades, diferenciando-se de um simples modelador 3D.

Relatos mais recentes sobre a inserção do ensino de BIM são apresentados por Menezes, Giesta e Baracho (2015), Santos et al. (2016) e Costa (2017) em cursos de Engenharia Civil e em curso técnico de Edificações. Algumas estratégias de introdução do BIM foram desenvolvidas por pesquisadores no Brasil. Checcucci (2014) sugere que o ensino de BIM seja inserido paulatinamente durante a formação do estudante, de forma a ir agregando crescente complexidade. Barison e Santos (2011b) destacam que o primeiro passo para implementar BIM em um currículo é saber quais são os níveis de proficiência em BIM que um aluno deve alcançar, ou seja, identificar competências BIM e determinar como e quanto tempo deve ser gasto no currículo para desenvolvê-las. Os níveis de proficiência podem ser: modelador BIM (nível introdutório); analista BIM (nível intermediário) e gerente BIM (nível avançado).

Menezes, Giesta e Baracho (2015) lembram que, hoje, grande parte dos discursos que tratam da reformulação dos currículos, visando à inserção da filosofia BIM, acontece dentro dos cursos de Arquitetura e Engenharia Civil, ficando de lado os cursos de Edificações, que são compostos por disciplinas básicas de Engenharia e Arquitetura. Além disso, a partir de pesquisa bibliográfica, os autores perceberam que poucas escolas técnicas têm se apropriado do BIM ou investido em pesquisas que tratem do tema, limitando-se ao treinamento de ferramentas do BIM. Em sua experiência no campus Natal do IFRN, os autores afirmam que dentro da atual estrutura curricular dos cursos de Edificações, não há espaço para inserir uma disciplina que seja baseada em BIM, mas que o BIM pode ser inserido na prática das disciplinas específicas.

Ao apresentar os resultados da inserção do BIM no curso técnico em Edificações do SENAI-SP, Cupperschmid, Cruz e Ruschel (2017) avaliaram que a inserção de técnicos qualificados para a modelagem básica da informação da construção na construção civil corrobora em tornar o mercado mais competitivo, favorecendo o crescimento do setor. Os autores verificaram a necessidade de transformar os cursos técnicos para inserir profissionais mais qualificados no mercado e permitir novas oportunidades de trabalho atendendo às novas demandas da indústria. Por isso, o impacto da inserção de BIM na formação de futuros profissionais para o mercado deve ser observado e analisado.

Trabalhos como de Barison e Santos (2011); Checcucci (2014); Menezes (2011) e Costa (2017) relataram algumas possíveis dificuldades no processo de implementação do BIM nos cursos de AEC: falta de tempo ou recursos para desenvolver um novo currículo; falta de espaço no currículo para incluir novas matérias; falta de materiais específicos para ensinar BIM; falta de infraestrutura para o ensino colaborativo e integrado de BIM, incluindo laboratórios de informática; falta de docentes com domínio na temática BIM; resistência cultural à mudança de paradigma; custo da tecnologia e a falta de bibliografia específica sobre o tema. 
Cupperschmid, Cruz e Ruschel (2017) dividem as dificuldades encontradas em três grupos: cultural, pedagógico e técnico, sendo que as maiores barreiras foram as pedagógicas (56\%), como por exemplo a falta de materiais didáticos $(24 \%)$ e de tempo necessário para amadurecer o currículo (16\%).

Ainda há pouco relatos sobre a introdução do BIM em cursos técnicos e tecnológicos no Brasil, sendo que as pesquisas sobre a inserção do BIM são predominantemente em cursos bacharelados de Arquitetura e Engenharia Civil. Por outro lado, existe uma preocupação quanto à introdução do BIM nos cursos cujos projetos político-pedagógicos ainda não contemplam uma metodologia específica para tal inserção.

Por meio de um estudo de caso no curso de Tecnologia em Construção de Edifícios do IFMT, esta pesquisa tem por objetivos: apresentar a percepção dos alunos quanto à introdução do BIM no curso, identificar as dificuldades na introdução do BIM na disciplina de Desenho Assistido por Computador e sugerir melhorias na metodologia de aplicação do BIM no curso.

\section{METODOLOGIA}

Para dar suporte à pesquisa utilizou-se o processo metodológico adaptado de GIL (2002), o levantamento, que se caracteriza pela interrogação direta das pessoas cujo comportamento se deseja conhecer.

O levantamento de dados abrangeu duas turmas do curso de Tecnologia em Construção de Edifícios cujos alunos foram matriculados na disciplina de Desenho Assistido por Computador durante os anos de 2018 e 2019.

Para conhecer as percepções dos alunos quanto aos desafios no aprendizado do BIM, um questionário foi elaborado com 13 perguntas objetivas e 01 pergunta aberta que buscavam investigar: o perfil social do aluno, o desempenho do aluno na disciplina cursada, a percepção do aluno quanto à importância do BIM para o mercado, as dificuldades de aprendizagem e sugestões de melhorias para implantação do BIM no curso.

Uma amostra de 26 alunos respondeu ao questionário e suas respostas foram analisadas segundo o conhecimento obtido com a revisão da literatura, completando a quarta etapa do processo metodológico.

\section{ANÁLISE E DISCUSSÃO DOS RESULTADOS}

Quanto à condição dos alunos de financiar um curso de software BIM, como o Revit, da empresa Autodesk, fora da escola, 87\% dos alunos responderam não ter condições. Essa pergunta confirma que os alunos dos cursos de Tecnologia são em sua grande maioria de baixa renda, corroborando a função social do Instituto Federal.

Cerca de $92 \%$ dos alunos consideram o BIM importante para o seu ingresso no mercado.

Foi questionado aos alunos sobre o seu desempenho na disciplina de Desenho Assistido por Computador, o qual foi usado o software Revit como ferramenta para modelagem da informação arquitetônica de um edifício. Conforme a Figura 1, 56\% deles consideram ter finalizado a disciplina com sucesso. Isto se confirma por meio da pergunta aberta do questionário "Como você define BIM?", pois percebe-se nas respostas que muitos alunos ainda entendem o BIM apenas como um programa ou ferramenta de desenho e não uma metodologia para o processo de projetar todo o ciclo de vida de uma edificação.

Para saber o impacto do aprendizado em BIM, 76\% dos alunos responderam que o BIM os ajudou a compreender conceitos e leituras de projetos. Além disso, se perguntou se o BIM tem auxiliado na compreensão de outras disciplinas, como tecnologia das construções, materiais de construções, instalações, estruturas, orçamento e planejamento. $60 \%$ dos alunos responderam que sim, como pode ser observado na Figura 2 . Isto 
demonstra que o BIM auxilia o aluno a compreender diferentes disciplinas, exatamente porque o BIM em sua definição é uma filosofia de trabalho que agrega diversas disciplinas de AEC. Um exemplo é caso de Costa (2017) que utilizou modelagem paramétrica como mediadora da construção do conhecimento em Desenho Técnico no curso de Edificações do IFBA, Campus Salvador. O autor relata que a aplicação da modelagem geométrica foi um instrumento capaz de desenvolver as habilidades de percepção das formas 3D e as habilidades para representação gráfica dos alunos do curso, além de ser útil para o ensino de elementos estruturais, elementos da cobertura, paredes, esquadrias, instalações, entre outros.

Figura 1 - Desempenho dos alunos no curso

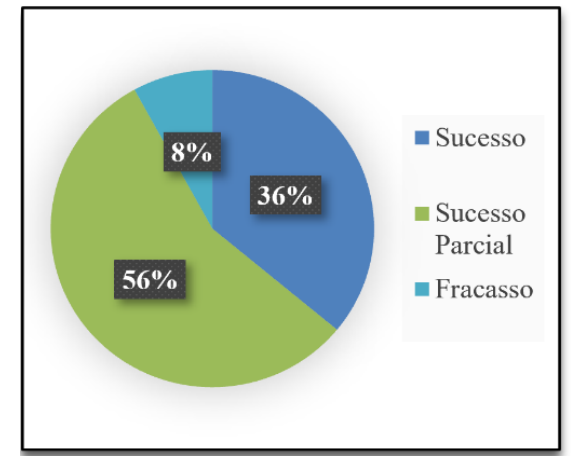

Fonte: Autoria própria
Figura 2 - O BIM como auxílio da compreensão de outras disciplinas

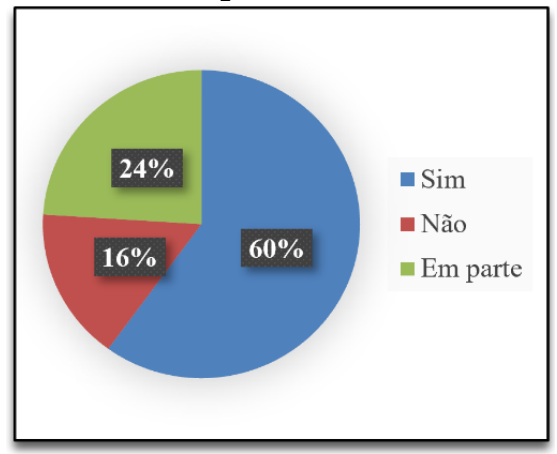

Fonte: Autoria própria

Sobre a continuação do ensino de BIM no curso, $96 \%$ responderam que o BIM deve continuar sendo implantado no curso e $100 \%$ confirmaram que o BIM deve continuar sendo utilizado nas disciplinas de projetos, planejamento e gerenciamento. Estas respostas revelam a aceitação dos alunos quanto ao ensino de BIM que tiveram contato com a metodologia dando abertura para os professores continuarem o ensino de BIM nas próximas disciplinas e turmas.

Para saber sobre as dificuldades e problemas no aprendizado do BIM, foram feitas duas perguntas. A primeira buscou saber quais os tópicos específicos do processo de modelagem que o aluno teve dificuldade, cujas respostas são apresentadas na Figura 3.

Figura 3 - Tópicos que o aluno teve mais dificuldade em aprender

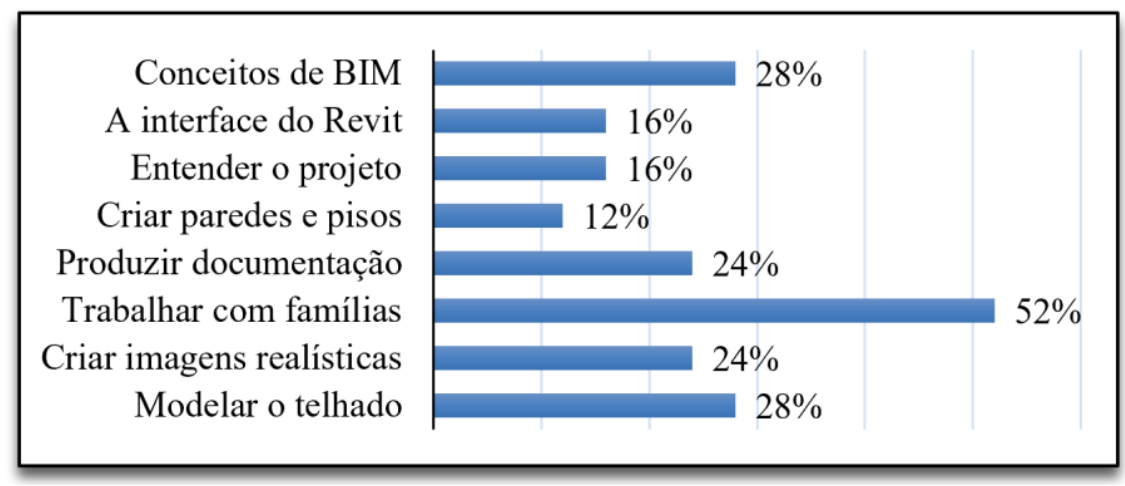

Fonte: Autoria própria

Quanto aos tópicos que os alunos mais tiveram dificuldade em aprender durante a disciplina de utilizando o software Revit, se destacam: trabalhar com famílias (52\%), conceitos BIM (28\%) e modelar o telhado (28\%). Sabe-se que o processo de modelagem de famílias e elementos da cobertura demandam tempo e dedicação. E, mais uma vez, percebe-se a dificuldade no entendimento dos conceitos de BIM, fortalecendo a necessidade de garantir uma sólida base conceitual de BIM. 
A segunda pergunta abrangeu os problemas, externos ou internos à escola, que o aluno encontrou durante todo o processo de aprendizagem do BIM. As respostas podem ser verificadas pela Figura 4.

\section{Figura 4 - Problemas que o aluno teve no processo de aprendizagem de um software BIM}

\begin{tabular}{|c|c|c|c|}
\hline \multirow{2}{*}{$\begin{array}{l}\text { Falta de tempo para praticar } \\
\text { Não ter computador próprio }\end{array}$} & & & \multirow[t]{2}{*}{$68 \%$} \\
\hline & & $36 \%$ & \\
\hline Espaço de tempo muito pequeno para a.. & 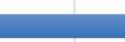 & $28 \%$ & \\
\hline Falta de conhecimento em informática básica & $8 \%$ & & \\
\hline Falta de conhecimento em construção civil & & $32 \%$ & \\
\hline Atendimento fora do horário de aula & $4 \%$ & & \\
\hline Infraestrutura do laboratório ineficiente & $4 \%$ & & \\
\hline Outros estudantes na sala de aula & $0 \%$ & & \\
\hline A interface do software utilizado & $0 \%$ & & \\
\hline Professor não conseguia atender todos em.. & $0 \%$ & & \\
\hline
\end{tabular}

Fonte: Autoria própria

Com relação aos problemas externos ou internos pode-se ressaltar: a falta de tempo para praticar e não ter computador próprio coincidem com o perfil social do aluno perceptível pela segunda pergunta. $\mathrm{O}$ espaço de tempo muito pequeno para a aprendizagem e a falta de conhecimento em construção civil indicam a necessidade da contemplação do ensino de BIM por outras disciplinas dando continuidade na aprendizagem e reforçando conceitos.

A última pergunta apurou as sugestões que poderiam ser dadas para melhorar o processo de ensino/aprendizagem do BIM no curso. A Figura 5 apresenta as respostas dadas pelos alunos.

Figura 5 - Sugestões do aluno para melhorar o processo de aprendizagem do BIM

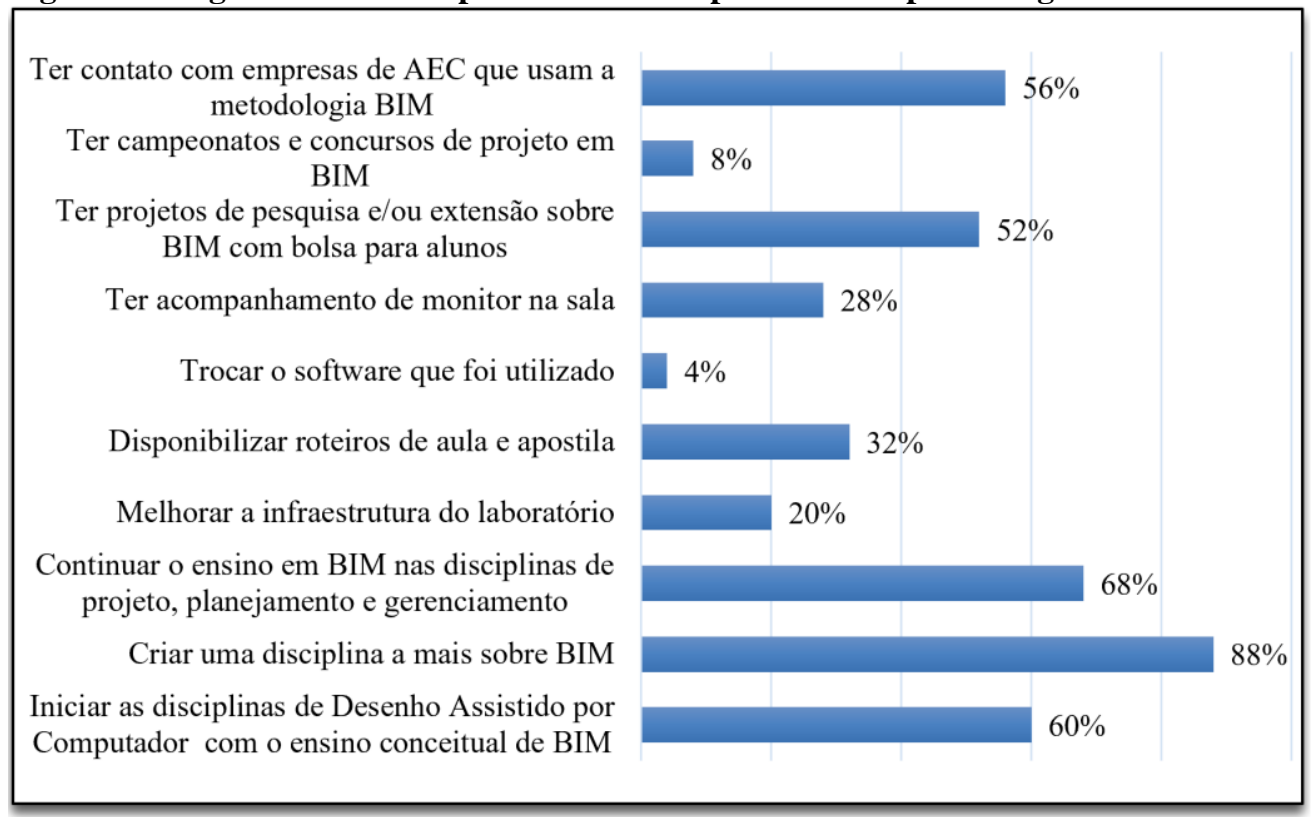

Fonte: Autoria própria

Acerca de criar uma disciplina a mais sobre BIM seria necessário analisar como poderia ser reformulada a matriz curricular do curso para que seja inserida uma disciplina específica sobre o ensino de BIM. Continuar o ensino de BIM nas disciplinas seguintes do curso reforça o interesse dos alunos em continuar aprendendo todos os aspectos do 
BIM e favorece o aprendizado contínuo desta metodologia. Novamente é apresentado pelos alunos o anseio pelo ensino dos conceitos do BIM, e a visualização da prática do BIM por meio do contato com empresas de AEC e mediante o desenvolvimento de projetos de pesquisa e extensão.

\section{CONSIDERAÇÕES FINAIS}

O Building Information Modeling (BIM) é um novo modo de projetar, o qual utiliza um grande volume de informação e necessita de um fluxo de colaboração entre profissionais de AEC que só foi possível pela evolução da tecnologia. A introdução do BIM no currículo de cursos que tratam de tecnologia das edificações como os tecnólogos em Construção de Edifícios de nível superior e os cursos de Engenharia Civil pode elevar a procura por estes profissionais, além de melhorar os índices de aproveitamento dos cursos.

Foram identificadas na bibliografia algumas estratégias para introdução do BIM, como: cursos de qualificação profissional (FIC), inserção de softwares BIM nas disciplinas de desenho, projetos de pesquisa envolvendo a tecnologia BIM e inclusive uma experiência gamificada do BIM (MENEZES; GIESTA; BARACHO, 2015).

A exemplo do estudo de caso desenvolvido, diversas ações estão sendo tomadas, como a capacitação de professores com cursos presenciais, minicursos para o público interno e externo da instituição sobre modelagem da informação arquitetônica, projetos de pesquisa, palestras para os alunos com empresas do ramo tratando sobre a importância do BIM e a inserção do conceito BIM e ferramentas para modelagem da informação na disciplina de Desenho Assistido por Computador.

Por meio da pergunta aberta do questionário "Como você define BIM?" percebe-se que muitos alunos ainda entendem o BIM apenas como um programa e ferramenta de desenho e não uma metodologia para o processo de projetar todo o ciclo de vida de uma edificação. Além disso, 28\% dos alunos responderam ter dificuldade em aprender sobre o conceito BIM, o que verifica a necessidade de fortalecer o ensino conceitual do que é o BIM.

Apesar disso, verificou-se que o uso do BIM tem facilitado o entendimento dos alunos em outras disciplinas. Outros casos já foram relatados que o uso do BIM pelos professores auxilia o aluno na compreensão da leitura de projetos, da dimensão 3D do edifício, da sequência da execução da obra e, por isso, seu emprego tem sido profícuo em diversos cursos de AEC.

Baseado na revisão da literatura, nas dificuldades reveladas pelos alunos e nas opções de sugestões escolhidas por eles, algumas propostas para melhoria da implantação do BIM no curso podem ser propostas:

1. Introdução do conceito de BIM em disciplinas básicas como o Desenho Técnico. Pode-se introduzir o ensino teórico sobre BIM, tratando também da suas aplicações e benefícios para a carreira. A base fornecida nessa disciplina sustentará a aplicação do BIM nas próximas disciplinas.

2. Uma proposta de disciplinas que podem ser utilizadas no processo de aprendizagem de BIM no curso do estudo de caso foi compilada na Figura 6. Nela podem ser visualizadas as disciplinas em destaque cujos professores podem ser capacitados e incentivados para utilização do BIM em um projeto único integrado. As disciplinas destacadas em vermelho podem trabalhar em um projeto único, produzindo a modelagem arquitetônica ainda no $2^{\circ}$ semestre e os projetos complementares nos semestres seguintes. No sexto semestre o aluno poderá compatibilizar esses projetos e será capacitado em gerenciar e coordenar projetos, formando um Gerente BIM do projeto. As disciplinas destacadas em azul podem utilizar modelagens paramétricas em BIM para apoio didático no ensino. 
3. Criação de um grupo de pesquisa que promova projetos de pesquisa e extensão sobre BIM. Como apresentado na revisão, um bom exemplo da implantação do BIM no ensino técnico é o caso do IFRN, o qual possui um grupo de pesquisa desde 2011 e tem sido uma alternativa enquanto não é possível inserir conteúdos BIM na grade curricular dos cursos existentes.

4. Promoção de palestras, workshops, seminários com empresas de AECO. Essa alternativa pode ser executada inclusive pelo grupo de pesquisa criado e os eventos podem potencializar a percepção dos alunos e professores da importância do BIM.

5. Criação de laboratórios exclusivos para o ensino de BIM e com monitores de sala em horários extraclasse. Se todos os professores envolvidos nas disciplinas destacadas na matriz curricular adotar o ensino de BIM nas suas disciplinas será necessário melhorar a infraestrutura existente. O próprio grupo de pesquisa a ser criado poderia ter o seu laboratório particular para o desenvolvimento das pesquisas. Os monitores em sala de aula são importantes para suprir a necessidade dos alunos que não possuem computador pessoal, dispõem de um tempo restrito e precisam de horários extras para praticar as ferramentas.

Figura 6 - Proposta de disciplinas que podem ser utilizadas no processo de aprendizagem de BIM no curso do estudo de caso

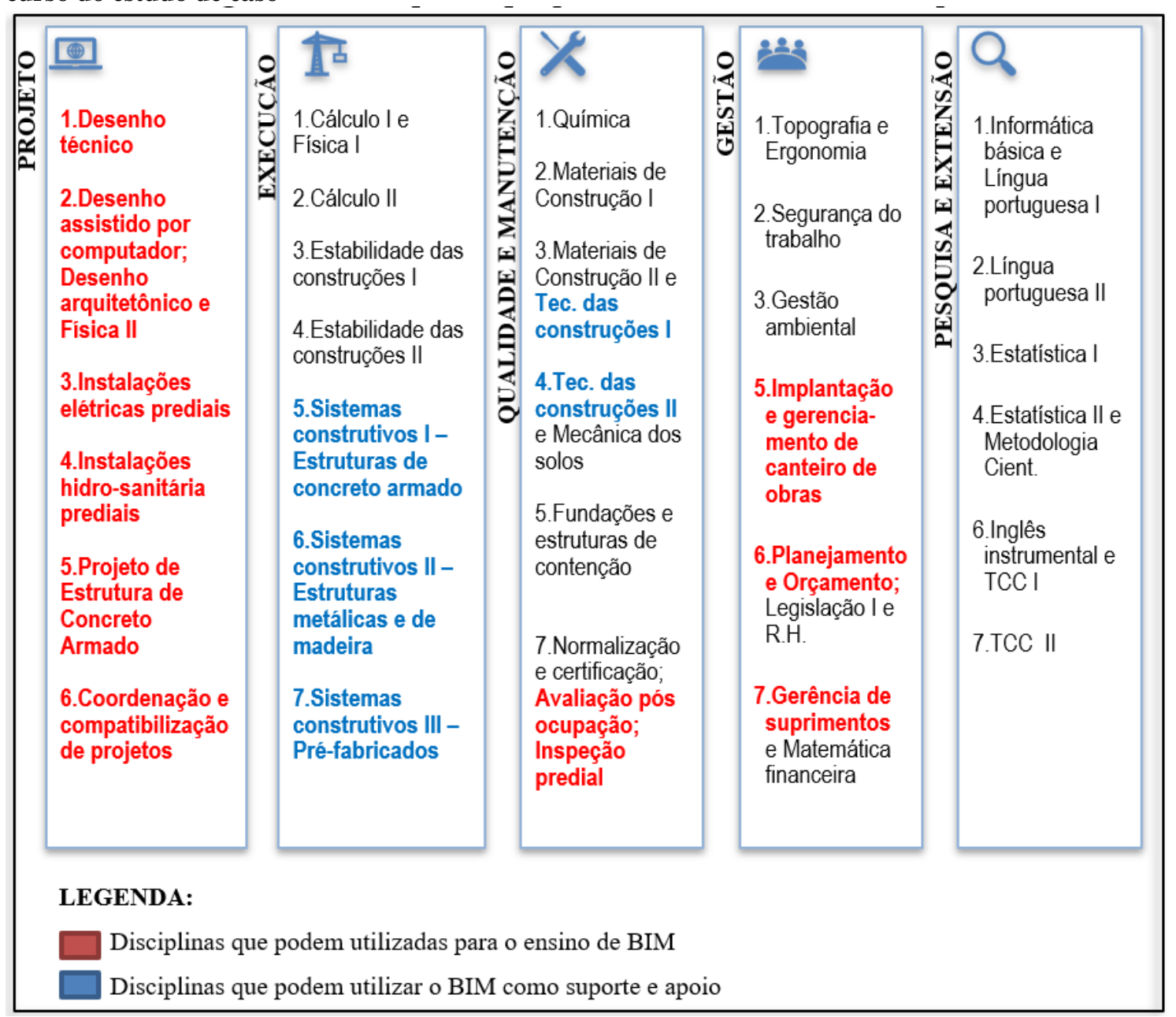

Fonte: Autoria própria

Por fim, essa pesquisa empenhou-se em registrar os desafios que tem sido a recente introdução do ensino de BIM no curso de Tecnologia e Construção de Edifícios do IFMT e a sua importância para a formação deste profissional. Os resultados apresentados podem contribuir estimulando o meio acadêmico a discutir a implantação e o aperfeiçoamento do processo de ensino-aprendizagem do Building Information Modeling ampliando as 
possibilidades de atuação profissional no contexto da Indústria 4.0 e como estratégia fundamental para o desenvolvimento tecnológico deste setor no país.

\section{REFERÊNCIAS}

BARISON, M. B.; SANTOS, E. T. Ensino de BIM: tendências atuais no cenário internacional. Gestão \& Tecnologia de Projetos, São Carlos, v. 6, n. 2, p. 67-80, dez. 2011.

CHECCUCCI, E. Ensino-aprendizagem de BIM nos cursos de graduação em Engenharia Civil. 2014. 235f. Tese (Doutorado em Difusão do Conhecimento) Faculdade de Educação da Universidade Federal da Bahia, Salvador.

COSTA, J. S. Modelegem geométrica como mediadora da construção do conhecimento em desenho técnico no curso de Edificações do IFBA, campus Salvador. 2017. 155f. Dissertação (Mestrado em Desenho, Cultura e Interatividade) Universidade Estadual de Feira de Santana, Feira de Santana.

CUPERSCHMID, A.; CRUZ, M. O.; RUSCHEL, R. C. A incorporação de BIM no ensino do curso Técnico em Edificações. Gestão \& Tecnologia de Projetos, São Carlos, v. 12, n. 2, p. 117-134, 2017.

GIL, A. C. Como Elaborar Projetos de Pesquisa. 4. ed. São Paulo: Atlas, 2002.

MENEZES, G. Breve histórico de implantação da plataforma BIM. Cadernos de Arquitetura e Urbanismo, v. 18, n. 22, 2011.

MENEZES, G. L. B. B.; GIESTA, J.; BARACHO, G.O. Uma experiência gamificada do BIM em cursos técnicos. In: CONGRESSO DE INICIAÇÃO CIENTÍFICA DO IFRN, XI., Santa Cruz, 2015. Anais...Santa Cruz, CONGIC/IFRN, 2015.

SANTOS, E. T.; FERREIRA, S. L.; CORRÊA, F. R.; LEITE, B. C. C.; BARISON, M. B. Relato de experiência de ensino de BIM em disciplina introdutória de curso de Engenharia Civil. In: ENCONTRO NACIONAL DE TECNOLOGIA DO AMBIENTE CONSTRUÍDO, XVI., São Paulo, 2016. Anais... São Paulo: ENTAC/USP, 2016. 